\title{
Modelling diffusion-reaction phenomena in yeast flocs of Saccharomyces cerevisiae
}

\author{
A.A. Vicente, M. Dluhý, E.C. Ferreira, J.A. Teixeira
}

Abstract Limitations in the diffusion of substrates into the flocs will condition cell metabolic behaviour, having obvious consequences on growth and product formation. Polymeric additives have been used aiming the reduction of those limitations. The knowledge of the concentration profiles and metabolic fluxes of glucose and oxygen inside the flocs would bring valuable information about the conditions under which a fermentation should run. Direct measurement of such profiles is rather difficult but their simulation has been performed and is presented in this work.

Calculations were made for different possible sizes of the yeast flocs, considering also the presence or absence of a polymeric additive. Only a small percentage of the cells in the flocs metabolise glucose oxidatively due to severe oxygen limitations. The presence of the polymeric additive increases the ratio of cells operating under respiratory metabolism over those under fermentative metabolism: from $0.4 \%$ to $5.7 \%$ without additive to $1.2 \%$ to $8.5 \%$ with additive, depending on the bulk glucose concentration. Also, based on this data, it is possible to justify the yeast floc natural shape.

\section{List of symbols}

a specific mass transfer area, $1 / \mathrm{m}$

$B i_{M}$ modified Biot number $\left(K_{c} \cdot R / D_{e, s}\right)$

$c$ concentration in floc, $\mathrm{g} / \mathrm{l}$

C concentration in bulk, $\mathrm{g} / \mathrm{l}$

$D$ dilution rate, $1 / \mathrm{s}$

$D_{e} \quad$ diffusion coefficient, $\mathrm{m}^{2} / \mathrm{s}$

$k_{L} \quad$ gas/liquid mass transfer coefficient for oxygen, $\mathrm{m} / \mathrm{s}$

$K \quad$ kinetic constant, $\mathrm{g} / \mathrm{l}$

$K_{c} \quad$ external mass transfer coefficient, $\mathrm{m} / \mathrm{s}$

$K_{i} \quad$ kinetic inhibition constant, g/l

Received: 15 May 1997

A.A. Vicente, M. Dluhý, E.C. Ferreira, J.A. Teixeira

Departamento de Engenharia Biológica,

Instituto de Biotecnologia e Química Fina,

Universidade do Minho, Campus de Gualtar,

4709 Braga codex, Portugal

Correspondence to: J.A. Teixeira

The financial support from the Sub-Programa Ciência e Tecnologia do $2^{\circ}$ Quadro Comunitário de Apoio through J.N.I.C.T. (Junta Nacional de Investigação Científica e Tecnológica) is gratefully acknowledged.
$K_{1}$ dimensionless kinetic saturation constant $\left(K_{S} / C_{S, F}\right)$

$K_{2}$ dimensionless kinetic saturation constant $\left(K_{O} / C_{S, F}\right)$

$q$

$r$

specific substrate consumption rate, $\mathrm{g} /\left(\mathrm{g}_{\text {biomass }} \cdot \mathrm{s}\right)$

radial floc coordinate, $\mathrm{m}$; reaction rate,

$\mathrm{g}_{\text {biomass }} /(1 \cdot \mathrm{s})$

floc radius, $\mathrm{m}$

time, $s$

temperature, $\mathrm{K}$

$Y_{X / E}$ biomass/ethanol yield coefficient

$Y_{X / O}$ biomass/oxygen yield coefficient

$Y_{X / S}$ biomass/substrate yield coefficient

\section{Greek letters}

$\alpha_{1} \quad$ modified external mass transfer coefficient for substrate $\left(K_{c, S} \cdot a \cdot \varepsilon_{p} / \varepsilon_{r}\right), 1 / \mathrm{s}$

$\alpha_{2} \quad$ modified external mass transfer coefficient for oxygen $\left(K_{c, O} \cdot a \cdot \varepsilon_{p} / \varepsilon_{r}\right), 1 / \mathrm{s}$

$\alpha_{3} \quad$ modified gas/liquid mass transfer coefficient for oxygen $\left(K_{L} \cdot a \cdot \varepsilon_{p} / \varepsilon_{r}\right), 1 / \mathrm{s}$

$\delta \quad$ dimensionless parameter $\left(D_{e, i} / D_{e, S}\right)$

$\varepsilon_{p} \quad$ floc porosity

$\varepsilon_{r} \quad$ void fraction

$\varphi$ dimensionless specific biomass growth rate

$\mu \quad$ total specific biomass growth rate

$\mathrm{g}_{\text {biomass }} /\left(\mathrm{g}_{\text {biomass }} \cdot \mathrm{s}\right)$

$v_{O} \quad$ stoichiometric coefficient for oxygen, ( $\mathrm{g}_{\text {oxygen }} / \mathrm{g}_{\text {glucose }}$ )

$\rho$ dimensionless radial coordinate in floc $(r / R)$

$\tau \quad$ dimensionless time $\left(t \cdot D_{e, S} /\left(\varepsilon_{p} \cdot R^{2}\right)\right)$

$\xi$ dimensionless concentration in floc $\left(c_{i} / C_{S, F}\right)$

$Z$ dimensionless concentration in bulk $\left(C_{i} / C_{S, F}\right)$

\section{superscripts}

add with flocculation additive

$f \quad$ fermentative

$r$ respiratory

sat saturation

subscripts

$E, E 1, E 2$ ethanol

$F \quad$ feed

$i \quad$ component

max maximum

$O \quad$ oxygen

$R \quad$ at floc surface

req required for respiration on glucose

$S \quad$ substrate

$X \quad$ biomass 


\section{1}

\section{Introduction}

One of the main goals of bioprocess engineering is to increase the productivity of biotechnological processes and there has been a vigorous progress in that field over the last decades. In order to achieve such objectives, techniques such as the use of high cell density systems have been thoroughly studied [1-5]. Among these, the use of flocculent micro-organisms cultivated in bioreactors where their sedimentation characteristics can be exploited is a very promising area, having low associated costs and design simplicity as main advantages $[6,7]$.

However, in order to take full advantage of such systems, their characterisation in terms of solutes' diffusional properties is an essential step, as diffusion is probably the most important mechanism of solute transport through cell aggregates [8-10].

Limitations in the diffusion of substrates will condition cell metabolic behaviour, being particularly critical the available amounts of the carbon source and oxygen [1114]. This will have obvious consequences on cell growth and product formation.

Attempts have been made in order to reduce diffusional limitations in flocs through the use of polymeric additives $[6,15,16]$. Yeast flocs form, according to Miki et al. [17] as "a specific lectin-like component of the cell wall recognises and adheres to $\alpha$-mannan carbohydrate on an adjoining cell, $\mathrm{Ca}^{2+}$ ions acting as a cofactor activating the binding capacity". Also ions such as $\mathrm{Mg}^{2+}, \mathrm{Co}^{2+}$ and $\mathrm{K}^{+}$promote flocculation while $\mathrm{La}^{3+}, \mathrm{Cr}^{2+}$ and others inhibit that process [18]. Further, fermentation medium composition can also influence surface properties and aggregation in yeast [19].

One way of enlarging the space between adjacent cells could be the use of additives that extend the bridges that link the cells in a floc [20]. In fact, reductions in diffusional limitations have been reported $[6,7,20,21]$, suggesting an increase of the effective diffusion coefficient of the substrates in the floc when some flocculating additives are used.

The values for the effective diffusivity of glucose and oxygen in yeast flocs with a flocculating additive have been determined using the technique developed by Vicente et al. [22]. Diffusivities without flocculating additive were already available from a previous work (submitted), using the same technique. Such data, together with an appropriate model and a suitable mathematical technique, allow the calculation of concentration profiles (of carbon source, oxygen) inside the objects of interest.

This procedure may be very helpful when trying to predict the metabolic behaviour of the aggregates, as well as it can afford some insight into floc structure and the effect of the additive.

The present work focuses on simulations of the concentration profiles of glucose and dissolved oxygen in yeast flocs during the course of a continuous fermentation. A model that includes a switch in the metabolism, depending on the respiratory bottleneck of Saccharomyces cerevisiae [14] has been developed (Appendix). The corresponding set of partial differential equations (PDE) was transformed in a set of ordinary differential equations
(ODE) by means of an orthogonal collocation method [23]. This system of ODE was finally solved by an appropriate integration routine, in order to obtain the concentration profiles. Calculations were made for different possible sizes of the yeast flocs, considering also the presence or absence of a flocculation additive (Magna Floc LT-25).

2 Model

\section{1}

\section{Assumptions of the model}

In order to develop a suitable model describing the system (yeast flocs in a continuous airlift reactor) in terms of mass balances of substrates both inside the flocs and in the bulk phase, several assumptions have been made as follows:

i) flocs are perfect spheres;

ii) there is no significant growth of biomass during the period of simulation;

iii) the floc population has one single diameter and has constant size during the period of simulation;

iv) the oxygen transfer rate from the gas to the liquid phase is not limiting;

v) in the beginning of the start up phase flocs are free from both glucose and oxygen;

vi) partition coefficients of substrates between medium and flocs are constant and equal to unity;

vii) metabolic reactions occur only inside the flocs; there is no reaction in the bulk liquid phase as there are no free suspended cells;

viii) diffusion and reaction in the flocs occur simultaneously;

ix) because of the lack of kinetic data for the yeast in the flocs, data from a free suspended cell fermentation [14] were used instead.

Further, it is assumed that the flocs have already been formed and are suspended in the fermentation broth containing all the necessary nutrients except the carbon source; the simulations start when medium containing glucose is fed in order to start the continuous fermentation at the chosen dilution rate. Oxygen is supplied continuously during the process.

\section{2}

\section{Model equations}

In order to develop the model, the system has been divided in two parts: Part A representing the flocs and Part B representing the bulk liquid phase. The coupling of both parts is made through the boundary conditions.

\section{Part A: flocs}

According to the well accepted metabolic model proposed by Sonnleitner and Käppeli [14], glucose can be consumed through a respiratory or a respiro-fermentative pathway. The pathway followed depends either on the availability of dissolved oxygen in the medium (if oxygen is limiting) or on the maximum respiratory capacity - the respiratory bottleneck - of the cells (if oxygen is not limiting). If either oxygen limitation occurs or maximum respiratory capacity is attained, the cells will consume glucose through respiro- 
fermentative metabolism. This is the base of the so-called switch model [14]. A short description of this model is made in Appendix.

The dimensionless specific growth rates for substrate for respiratory and fermentative metabolism are presented in Eq. (1) and (2), respectively:

$$
\begin{aligned}
\varphi_{S}^{r}= & \left(\frac{R^{2}}{D_{e, S}}\right) \cdot Y_{X / O}^{r} \cdot q_{O, \max } \cdot \frac{\xi_{O}}{K_{2}+\xi_{O}}, \\
\varphi_{S}^{f}= & \left(\frac{R^{2}}{D_{e, S}}\right) \cdot Y_{X / S}^{f} \cdot\left(q_{S, \max } \cdot \frac{\xi_{S}}{K_{1}+\xi_{S}}\right. \\
& \left.-q_{O, \max } \cdot \frac{Y_{X / O}^{r}}{Y_{X / S}^{r}} \cdot \frac{\xi_{O}}{K_{2}+\xi_{O}}\right) .
\end{aligned}
$$

These two variables are the reaction terms in the dimensionless mass balance equations for glucose and oxygen, Eq. (3) and (4) respectively:

$$
\begin{aligned}
\frac{\partial \xi_{S}}{\partial \tau}= & \delta_{S} \cdot\left(\frac{\partial^{2} \xi_{S}}{\partial \rho^{2}}+\frac{2}{\rho} \cdot \frac{\partial \xi_{S}}{\partial \rho}\right) \\
& -\left(\frac{\varphi_{S}^{r}}{Y_{X / S}^{r}}+\frac{\varphi_{S}^{f}}{Y_{X / S}^{f}}\right) \cdot Z_{X} \\
\frac{\partial \xi_{O}}{\partial \tau}= & \delta_{O} \cdot\left(\frac{\partial^{2} \xi_{O}}{\partial \rho^{2}}+\frac{2}{\rho} \cdot \frac{\partial \xi_{O}}{\partial \rho}\right) \\
& -\frac{\varphi_{S}^{r}}{Y_{X / O}^{r}} \cdot Z_{X}
\end{aligned}
$$

with the following initial and boundary conditions:

$$
\begin{array}{ll}
\tau \leq 0 & 0<\rho<1 \quad \xi_{i}=0, \\
\tau>0 & \rho=0 \quad \frac{\partial \xi_{i}}{\partial \rho}=0, \\
\rho=1 & \frac{\partial \xi_{i}}{\partial \rho}=B i_{M, i} \cdot\left(Z_{i}-\xi_{i}\right), \text { for } i=S \text { or } O .
\end{array}
$$

\section{Part B: bulk phase}

For the bulk liquid phase, the dimensionless mass balances for glucose and oxygen are represented by Eq. (8) and (9), respectively:

$$
\begin{aligned}
& \frac{\mathrm{d} Z_{S}}{\mathrm{~d} \tau}=-\frac{R^{2}}{D_{e, S}} \cdot\left[\alpha_{1} \cdot\left(Z_{S}-\xi_{S, R}\right)-D \cdot\left(Z_{S, F}-Z_{S}\right)\right] \\
& \frac{\mathrm{d} Z_{O}}{\mathrm{~d} \tau}=-\frac{R^{2}}{D_{e, S}} \cdot\left[\alpha_{2} \cdot\left(Z_{O}-\xi_{O, R}\right)-\alpha_{3} \cdot\left(Z_{O}^{\text {sat }}-Z_{O}\right)\right]
\end{aligned}
$$

The option of normalising the above set of equations - (1) to (9) - enabled the attainment of a formally much simpler mathematical model, with a significantly reduced number of parameters; further, it allowed the use of the orthogonal collocation method in the subsequent calculations.

\section{3}

\section{Solution of the model equations}

As a first step to solve the model, Eqs. (1) to (7) need to be converted from PDE to ODE. This is achieved employing an orthogonal collocation technique (Villadsen and Michelsen, [23]). This technique is based on the idea that the space derivatives of the substrate concentrations are discretised by the sum of the weighted concentrations in the collocation points. These are the roots of the non-symmetric orthogonal Legendre polynomial (node polynomial). Seventeen internal collocation points were distributed along the radial coordinate of the floc, with an increasing density from the centre to the surface, where an eighteenth point has been placed. The collocation weights have been calculated by means of zeros of the node polynomial and the values of its first two derivatives in the zeros.

Once Eqs. (1) to (7) have been converted to ODE, the whole system (Eqs. (1) to (9)) has been integrated using Merson's modification of the Runge-Kutta method of fourth order with a non-constant integration step. This method allows an efficient integration of the so-called stiff systems such as the present one.

\section{4}

\section{Values of the model parameters}

The values of the effective diffusion coefficients for glucose and oxygen in the presence of a polymeric additive (Magna Floc LT-25, here designated by MF, in the concentration of $0.01 \% \mathrm{w} / \mathrm{v}$ ) have been determined using the method described by Vicente et al. [22]; the corresponding values in the absence of additive were the subject of a previous work (submitted).

Data concerning the bulk concentration versus time profiles for both glucose and dissolved oxygen have been treated by a numerical approach based on Fick's law of diffusion, solved by the orthogonal collocation method of Villadsen and Michelsen [23] with an integration routine of Runge-Kutta of the $4^{\text {th }}$ order with an automatic step control, coupled with a suitable optimisation technique, very much resembling the one that has been used for the simulations in the present work. Due to the oscillatory characteristics of the dissolved oxygen probe response, data were previously smoothed by a Fast Fourier Transformation technique.

Values of the effective diffusion coefficients, together with the values of the model parameters applied in the simulations, are listed in Tables 1a and $1 \mathrm{~b}$. Those were

Table 1a. Values of the model parameters held constant in the simulations

\begin{tabular}{llll}
\hline Parameter value & Parameter & value \\
\hline$D_{e, O}$ & $2.49 \times 10^{-11} \mathrm{~m}^{2} / \mathrm{s}^{\mathrm{a}}$ & $Y_{X / \mathrm{S}}^{f}$ & $0.05^{\mathrm{c}}$ \\
$D_{e, O}^{\text {add }}$ & $2.20 \times 10^{-10} \mathrm{~m}^{2} / \mathrm{s}^{\mathrm{b}}$ & $Y_{X / S}^{r}$ & $0.49^{\mathrm{c}}$ \\
$D_{e, S}$ & $1.10 \times 10^{-10} \mathrm{~m}^{2} / \mathrm{s}^{\mathrm{a}}$ & $C_{O}^{\text {sat }}$ & $0.008 \mathrm{~g} / \mathrm{l}$ \\
$D_{e, S}^{\text {add }}$ & $4.32 \times 10^{-10} \mathrm{~m}^{2} / \mathrm{s}^{\mathrm{b}}$ & $C_{X}$ & $5.0 \mathrm{~g} / \mathrm{l}$ \\
$K_{O}$ & $1.0 \times 10^{-4} \mathrm{~g} / \mathrm{l}^{\mathrm{c}}$ & $D$ & $5.6 \times 10^{-5} 1 / \mathrm{s}$ \\
$K_{S}$ & $0.2 \mathrm{~g} / \mathrm{l}^{\mathrm{c}}$ & $\varepsilon_{p}$ & $0.5^{\mathrm{d}}$ \\
$q_{O, \max }$ & $7.11 \times 10^{-5} \mathrm{~g} /\left(\mathrm{g}_{\text {biomass }} \cdot \mathrm{s}\right)^{\mathrm{c}}$ & $\varepsilon_{p}^{\text {add }}$ & $0.6^{\mathrm{d}}$ \\
$q_{S, \max }^{\mathrm{c}}$ & $9.72 \times 10^{-4} \mathrm{~g} /\left(\mathrm{g}_{\text {biomass }} \cdot \mathrm{s}\right)^{\mathrm{c}}$ & $T$ & $303 \mathrm{~K}$ \\
$Y_{X / O}^{r}$ & $1.20^{\mathrm{c}}$ & & \\
\hline
\end{tabular}

${ }^{a}$ unpublished data, submitted, ${ }^{b}$ this work, ${ }^{\mathrm{c}}$ Ref. [14], ${ }^{\mathrm{d}}$ Ref. [20] 
Table 1b. Values of the model parameters changed in the simulations

\begin{tabular}{llll}
\hline Parameter values & \multicolumn{2}{l}{ Parameter } & values \\
\hline$C_{O}$ & $10 ; 100 \%$ sat & $R$ & $0.45 ; 0.80 ; 1.20 \mathrm{~mm}$ \\
$C_{S}$ & $0.1 ; 0.5 ; 1 ; 10 ; 100 \mathrm{~g} / \mathrm{l}$ & & \\
\hline
\end{tabular}

used to obtain the values of the dimensionless groups present in the model equations (Eqs. 1 to 9). In the simulations several parameters were held constant (Table 1a) and others were changed (Table $1 \mathrm{~b}$ ) in order to investigate their influence on the glucose and dissolved oxygen concentration profiles in the flocs.

\section{3}

\section{Results and discussion}

\section{1}

\section{Simulations without flocculation additive}

Once the steady state is achieved, the concentration profiles are not expected to change and can give some interesting insight into the behaviour of the system. Such profiles are plotted in Figs. 1 to 3 for different floc radii $(0.45,0.80$ and $1.20 \mathrm{~mm}$, respectively).

In Figs. 1a, 2a and 3a the profiles for glucose at different bulk liquid concentrations are compared, while ranging from 0.1 to $100 \mathrm{~g} / \mathrm{l}$. The parameters near the lines are the values of the bulk glucose concentration to which each line corresponds. In a real system, this values can be achieved either through a significantly higher feed concentration (if the dilution rate is low) or through a significantly higher dilution rate (if the concentration in the feed is similar to that of the bulk). Dimensionless variables are displayed in order to allow a comparison of the simulations with different concentrations.

As clearly shown, glucose does not reach the centre in significant amounts, for bulk concentrations of $1 \mathrm{~g} / \mathrm{l}$ or less, typically found in continuous fermentations. Therefore, in a part of the floc volume glucose is not available to the cells. This situation is aggravated by the increase of the floc size: while for a floc with $0.45 \mathrm{~mm}$ of radius all the volume is reached by glucose, for a floc with a radius of $1.20 \mathrm{~mm}$, that substrate is present in only around $88 \%$ of the total volume. For higher bulk concentrations glucose does reach the centre of the aggregates.

Figures $1 \mathrm{~b}, 2 \mathrm{~b}$ and $3 \mathrm{~b}$ show the dissolved oxygen concentration profiles for the same situations described above. With bulk glucose concentrations of $0.1,0.5,1$ and $10 \mathrm{~g} / \mathrm{l}$, $10 \%$ of the saturation concentration of oxygen in water was set in the bulk and the respective profiles in flocs correspond to the solid line; in fact, all the profiles practically coincide so only one line has been represented for clarity (solid line). With a bulk glucose concentration of $100 \mathrm{~g} / \mathrm{l}, 100 \%$ of the saturation concentration of oxygen in water was set in the bulk and the corresponding profile in flocs is represented by the dashed line.

In all cases the penetration depth of the oxygen depends on the bulk concentration. It is, nevertheless and also in all cases, quite small, corresponding to small volumes of the floc (Table 2).
As expected, as the same value for the oxygen diffusivity was used in all simulations, the penetration depth is also constant. On the contrary, the volumetric percentage of the floc where dissolved oxygen is present diminishes significantly as the size of the aggregate increases.

Comparing the data calculated in this work with that presented by Sousa et al. [24], at least four fold higher values were obtained in the first case. The differences in the method used to assess the penetration depth are acute, making a comparison difficult. Nevertheless, data are of the same order of magnitude and other works on the subject could not be found.

Figures 1 to 3 show clearly that while oxygen is rapidly depleted in a narrow shell beneath the surface of the flocs, glucose still exists in interior parts. This is true even for low bulk glucose concentrations (around $1 \mathrm{~g} / \mathrm{l}$ and less) such as those commonly found in continuous fermentations. This has obvious implications on the biomass growth and mainly on ethanol production, according to
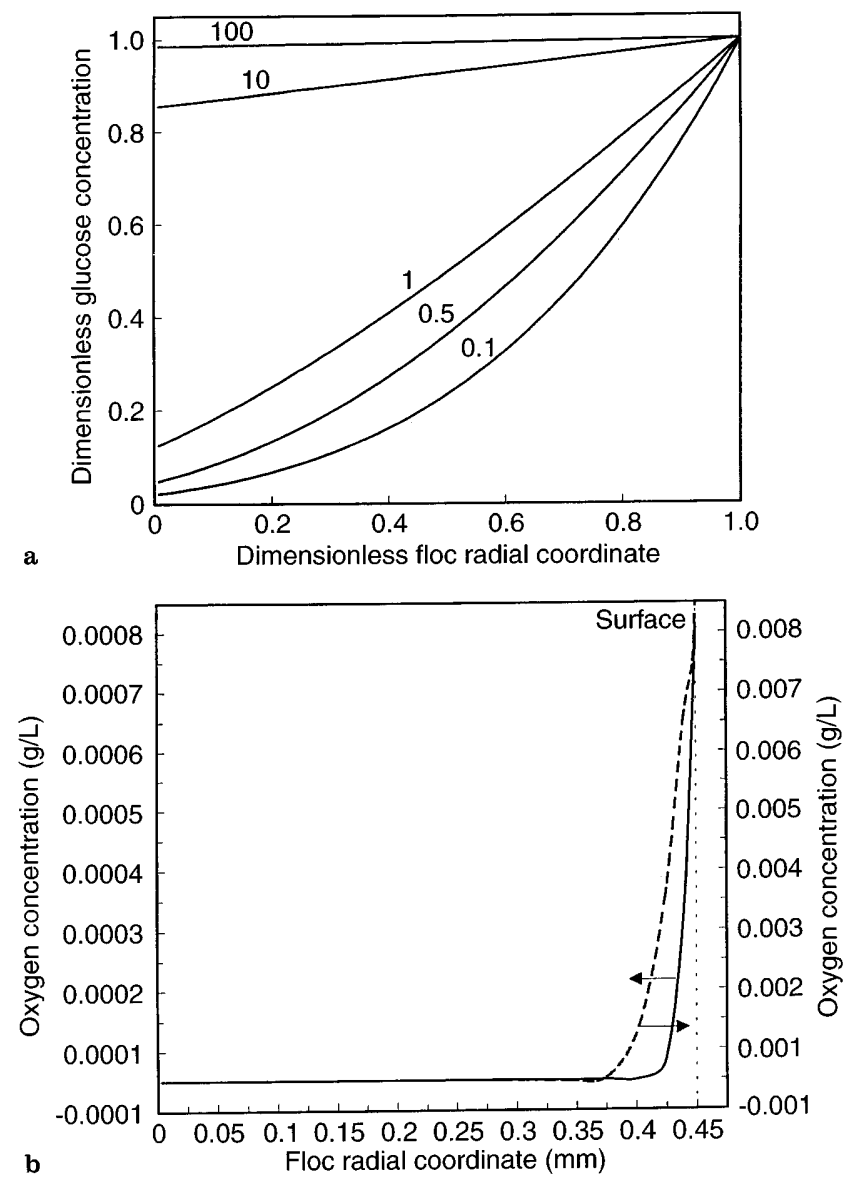

Fig. 1a,b. Concentration profiles for flocs with a radius of $0.45 \mathrm{~mm}$ : a dimensionless glucose concentration profiles; the parameters near the lines identify the values of the bulk concentration to which each line corresponds; $\mathbf{b}$ dissolved oxygen concentration profiles; the solid line represents all the profiles calculated for $10 \%$ (corresponding to $0.1,0.5,1$ and $10 \mathrm{~g} / \mathrm{l}$ of glucose) and the dashed line represents the profiles calculated for $100 \%$ (corresponding to $100 \mathrm{~g} / \mathrm{l}$ of glucose) of the oxygen saturation concentration in water 
the respiratory bottleneck model of Sonnleitner and Käppeli [14].

The oscillating/negative values of dissolved oxygen concentration are exclusively due to the calculation method applied in this work, making use of orthogonal collocation weights.

According to previous experimental knowledge, yeast floc's geometrical shape is very much dependent on their size; concretely, flocs with radii around $0.45 \mathrm{~mm}$ are spherical but an increase in size induces a gradual change in shape for that of a red blood cell: a flat cylinder thicker on the edges and thinner in the centre. This very interesting observation can be now justified with the results of the present work. As can be observed in Fig. 1a, there are not very significant glucose limitations in the centre of the floc. Nevertheless, the situation changes when floc size increases and that is very clear from Figs. $2 a$ and $3 a$. If the flocs were spherical (as assumed when simulating the concentration profiles) severe substrate limitations would occur in the central part of the aggregates. Being so, they

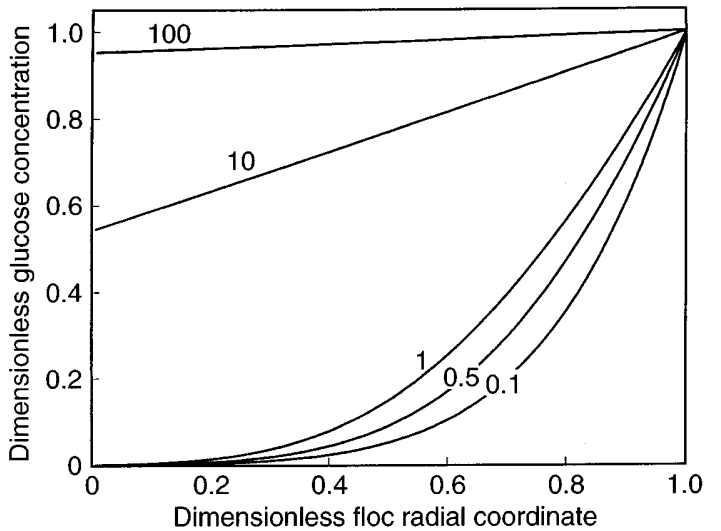

a

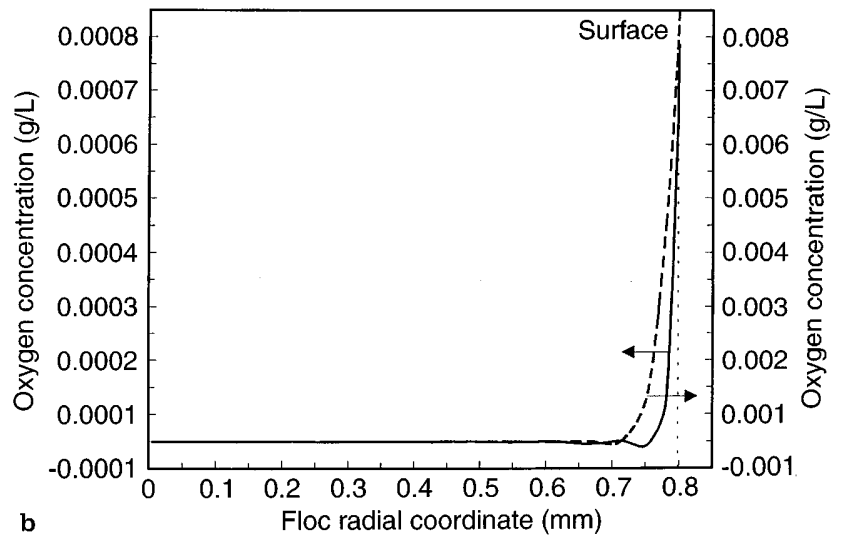

Fig. 2a,b. Concentration profiles for flocs with a radius of $0.80 \mathrm{~mm}$ : a dimensionless glucose concentration profiles; the parameters near the lines identify the values of the bulk concentration to which each line corresponds; b dissolved oxygen concentration profiles; the solid line represents all the profiles calculated for $10 \%$ (corresponding to $0.1,0.5,1$ and $10 \mathrm{~g} / \mathrm{l}$ of glucose) and the dashed line represents the profiles calculated for $100 \%$ (corresponding to $100 \mathrm{~g} / \mathrm{l}$ of glucose) of the oxygen saturation concentration in water
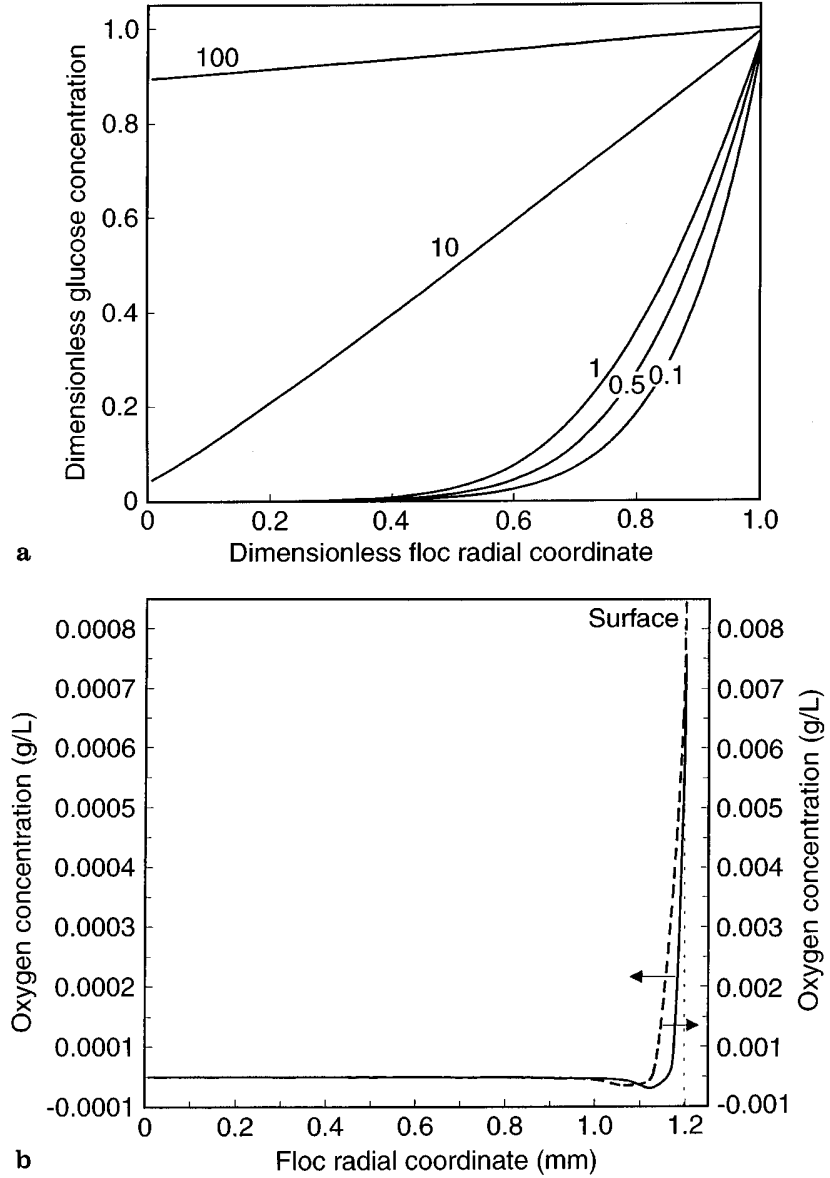

Fig. 3a,b. Concentration profiles for flocs with a radius of $1.20 \mathrm{~mm}$ : a dimensionless glucose concentration profiles; the parameters near the lines identify the values of the bulk concentration to which each line corresponds; $\mathbf{b}$ dissolved oxygen concentration profiles; the solid line represents all the profiles calculated for $10 \%$ (corresponding to $0.1,0.5,1$ and $10 \mathrm{~g} / \mathrm{l}$ of glucose) and the dashed line represents the profiles calculated for $100 \%$ (corresponding to $100 \mathrm{~g} / \mathrm{l}$ of glucose) of the oxygen saturation concentration in water

are actually not spherical but have the above mentioned red blood cell shape in which such limitations are reduced.

On the other hand, also the hydrodynamic characteristics of the bioreactor where the process takes place play an important role, as it is not possible to obtain flocs with the mentioned geometrical shapes if too large shear stresses are present (e.g. if a stirred tank bioreactor is used); rather, an airlift bioreactor is needed, where those stresses are significantly lower [25].

\section{2}

\section{Simulations with flocculation additive}

Figure 4 presents the profiles for glucose at different bulk liquid concentrations, for flocs with $0.8 \mathrm{~mm}$ of diameter and grown in the presence (dot-dashed lines) and absence (solid lines) of MF.

It is clearly seen that a significant increase in the glucose concentration in the flocs is achieved when MF is present, for the same bulk sugar concentrations. This is caused by a 
$20 \%$ increase of the floc porosity [20], together with a predictable alteration of pore size and structure. In fact, there is a four fold increase in the value of the effective diffusion coefficient of glucose in the flocs. This will lead to a deeper penetration of the sugar in the floc, as the glucose consumption rate is supposed to be constant and at its maximum value when a steady state condition is achieved. Nevertheless, glucose consumption will be affected by the availability of dissolved oxygen [14], which has also been changed because of the presence of MF.

In Fig. $4 \mathrm{~b}$ dissolved oxygen concentration profiles in the flocs are compared for the studied situations: with (dotdashed line) and without (solid line) MF, with a bulk dissolved oxygen concentration corresponding to $10 \%$ of saturation. As stated above for the case of glucose, also a very significant difference has been found between the profiles in question. As in the former case, and by the same reasons, there was an increase in the value of the effective diffusion coefficient of oxygen in the flocs (eight fold). The penetration depth of dissolved oxygen in the floc increased almost three fold and the corresponding percentage of the total floc volume with available dissolved oxygen rose to around $41 \%$ in the presence of MF, while without the additive dissolved oxygen is available only in $17 \%$ of the total floc volume (for flocs with the same size).

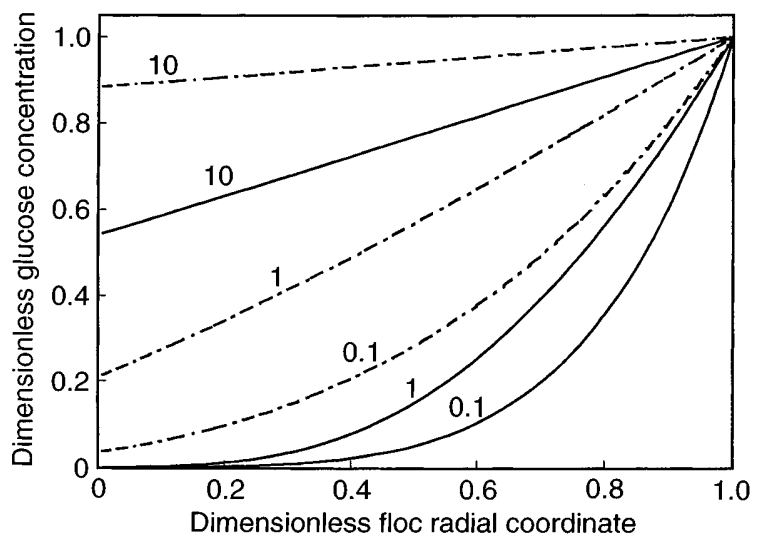

a

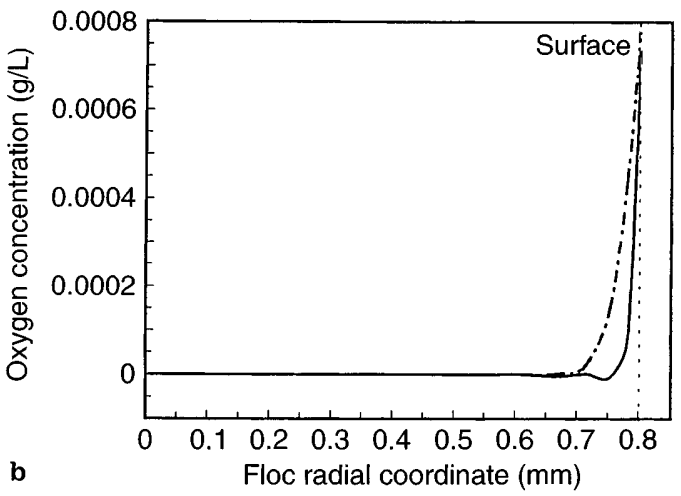

Fig. 4a,b Comparison of the concentration profiles for flocs with (dot-dashed lines) and without (solid lines) a flocculation additive, with a radius of $0.80 \mathrm{~mm}$ : a dimensionless glucose concentration profiles; the parameters near the lines identify the values of the bulk concentration to which each line corresponds; b dissolved oxygen concentration profiles

\section{3}

\section{Metabolism in the flocs}

Figures 5 and 6 represent the metabolic fluxes along the floc radial coordinate, for flocs with a radius of $0.8 \mathrm{~mm}$ grown without and with MF, respectively. The shaded area corresponds to the relative amount of cells growing under respiratory metabolism, as the curve that limits it represents the dissolved oxygen flux along the floc radial coordinate. The open area between each of the labelled curves (the labels refer to the respective bulk glucose concentrations) and the dissolved oxygen flux curve corresponds to the relative amount of cells growing under fermentative metabolism for each bulk glucose concentration. These statements are valid only for a regular distribution of cells in the floc. Further, the correspondence made between area and relative amount of cells is valid only because the metabolic flux curves have been calculated taking into account the respective stoichiometric coefficients of glucose and oxygen as used in the kinetic model described in Appendix.

The dashed line indicates the dissolved oxygen penetration depth as previously determined in Table 2 .

Table 2. Comparison of the penetration depths of dissolved oxygen in flocs with different radii and the corresponding $\%$ of floc volume

\begin{tabular}{llll}
\hline $\begin{array}{l}\text { Floc } \\
\text { radius } \\
(\mathrm{mm})\end{array}$ & $\begin{array}{l}\text { Penetration depth } \\
(\mu \mathrm{m}) \\
(10 \% \text { sat-100\% sat })\end{array}$ & $\begin{array}{l}\text { \% of total volume } \\
(10 \% \text { sat-100\% sat })\end{array}$ & Reference \\
\hline 0.45 & $47-75$ & $28.2-42.1$ & this work \\
0.80 & $47-75$ & $16.6-25.6$ & this work \\
$0.80^{\mathrm{a}}$ & $130-^{\mathrm{b}}$ & $41.3-^{\mathrm{b}}$ & this work \\
1.20 & $47-75$ & $11.3^{-17.6}$ & this work \\
0.45 & 13 & $8.3^{\mathrm{c}}$ & {$[24]$} \\
0.80 & 11 & $4.1^{\mathrm{d}}$ & {$[24]$} \\
\hline
\end{tabular}

${ }^{\mathrm{a}}$ with flocculation additive, ${ }^{\mathrm{b}}$ not calculated, ${ }^{\mathrm{c}} 41 \%$ sat by pure $\mathrm{O}_{2},{ }^{\mathrm{d}} 6.3 \%$ sat by pure $\mathrm{O}_{2}$

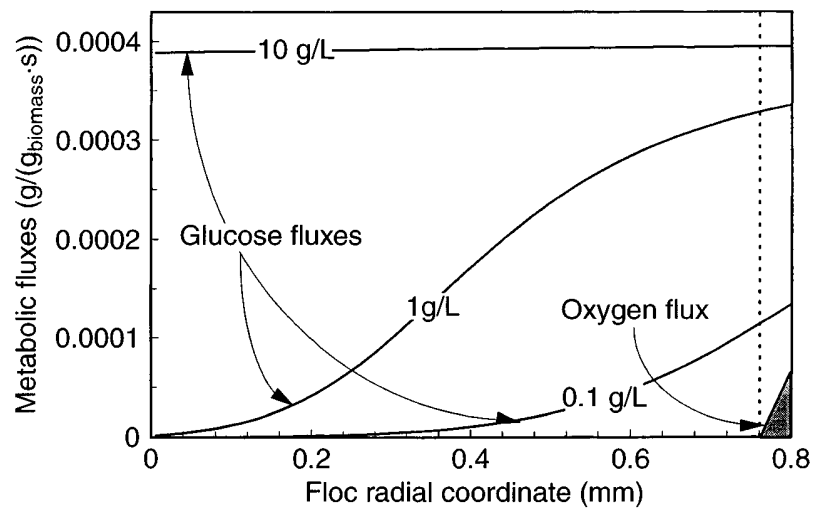

Fig. 5. Metabolic fluxes along the floc radial coordinate for flocs grown without MF. The shaded area corresponds to the relative amount of cells growing under respiratory metabolism and the open area between the labelled curves and the shaded area corresponds to the relative amount of cells growing under fermentative conditions. The numbers near the curves refer to the bulk glucose concentration. The dashed line corresponds to the dissolved oxygen penetration depth 


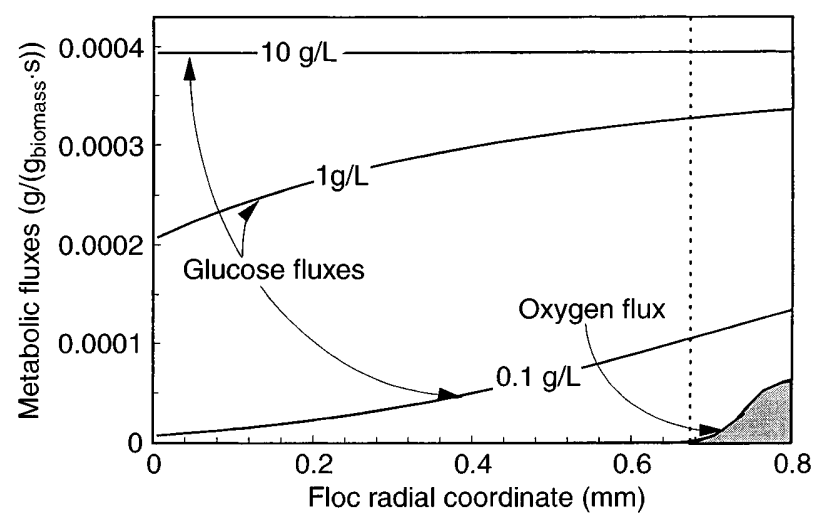

Fig. 6. Metabolic fluxes along the floc radial coordinate for flocs grown with MF. The shaded area corresponds to the relative amount of cells growing under respiratory metabolism and the open area between the labelled curves and the shaded area corresponds to the relative amount of cells growing under fermentative conditions. The numbers near the curves refer to the bulk glucose concentration. The dashed line corresponds to the dissolved oxygen penetration depth

As outlined before, only a small amount of cells, immediately below the floc surface, are engaged in respiratory consumption of glucose, while the majority metabolises glucose under fermentative conditions. There is no free dissolved oxygen capacity for oxidative ethanol consumption.

Comparing Figs. 5 and 6 it is clearly seen that the presence of MF increases the ratio of cells operating under respiratory metabolism over those under fermentative metabolism: from $0.4 \%$ to $5.7 \%$ without MF to $1.2 \%$ to $8.5 \%$ with that additive, according to bulk glucose concentration.

\section{4}

\section{Conclusions}

Despite the assumptions made, it is possible to conclude that there are strong substrate diffusional limitations in yeast flocs. These limitations influence the metabolic responses of the microorganisms and will affect the overall behaviour of a fermentation system. The results obtained from the simulations allowed for a partial understanding and justification of the yeast floc natural shape. The polymeric flocculation additive Magna Floc LT-25 showed to have a positive effect on the mass transfer properties as its presence was reflected in the higher values of effective diffusion coefficients for glucose (four fold) and oxygen (eight fold) when compared to those in its absence.

Only a small percentage of the cells in the floc metabolise glucose oxidatively due to severe oxygen limitations. Consequently, there is no oxidative ethanol consumption under the studied conditions.

It is possible, through the use (or not) of a polymeric additive such as Magna Floc LT-25, to shift the metabolism towards biomass or ethanol production.

\section{Appendix}

The present work uses the kinetic model proposed by Sonnleitner and Käppeli [14], based on the concept of respiratory bottleneck.
The cell growth on glucose with ethanol production/ consumption can be characterised by three major metabolic pathways: a) respiratory growth on glucose; b) fermentative growth on glucose and c) respiratory growth on ethanol.

The cells are supposed to have a limited respiratory capacity, i.e., a respiratory bottleneck. If the total amount of substrate(s) flux can pass the bottleneck (using pathways a) and/or c)) substrate flux is named subcritical; when the bottleneck is completely filled (but not exceeded), substrate flux is critical; if substrate(s) flux exceeds the bottleneck, then it is named supracritical. The excess is metabolised through pathway b) (except in the case of oxidative growth on ethanol).

Both the specific (total) glucose consumption rate and the specific oxygen uptake rate can be represented by Monod's kinetic model:

$q_{S}=q_{S, \max } \cdot \frac{C_{S}}{K_{S}+C_{S}}$,
$q_{O}=q_{O, \max } \cdot \frac{C_{O}}{K_{O}+C_{O}}$,

Biomass growth is an autocatalytic reaction:

$r_{X}=\mu \cdot C_{X}$.

The amount of dissolved oxygen required for respiration of glucose is defined as:

$q_{O, \text { req }}=\frac{Y_{X / S}^{r}}{Y_{X / O}^{r}} \cdot q_{s}$.

Depending on the availability of dissolved oxygen in the medium, two situations can occur (switch model):

1) $q_{O \text {,req }} \leq q_{O}$ (subcritical/critical substrate flux). Essentially all glucose is metabolised oxidatively. The residual respiratory capacity can be utilised for ethanol growth if it is present in the medium. Being so, the specific biomass growth rate is the sum of the following contributions:

$\mu_{S}^{r}=Y_{X / S}^{r} \cdot q_{s}$,

$\mu_{S}^{f}=0$.

Ethanol uptake also follows Monod kinetics and the priority of glucose uptake over ethanol uptake can be represented as an inhibition of the latter by glucose:

$\mu_{E 1}^{r}=\mu_{E, \max } \cdot \frac{C_{E}}{K_{E}+C_{E}} \cdot \frac{K_{i}}{K_{i}+C_{S}}$.

The remaining respiratory capacity $\left(q_{O}-q_{O, \text { req }}\right)$ is used in pathway c), for biomass production:

$\mu_{E 2}^{r}=\frac{Y_{X / O, E}^{r}}{Y_{X / E, E}^{r}} \cdot\left(q_{O}-q_{O, \text { req }}\right)$.

Consequently, $\mu_{E 1}^{r} \leq \mu_{E 2}^{r}$ and therefore the specific biomass growth rate on ethanol $\left(\mu_{E}^{r}\right)$ is considered to be: 


$$
\mu_{E}^{r}=\min \left(\mu_{E 1}^{r}, \mu_{E 2}^{r}\right)
$$

2) $q_{O, \text { req }}>q_{O}$ (supracritical substrate flux). All the respiratory capacity is used to metabolise oxidatively glucose according to pathway a):

$$
\mu_{S}^{r}=Y_{X / S}^{r} \cdot q_{O} \cdot \frac{Y_{X / O}^{r}}{Y_{X / S}^{r}},
$$

and the remaining glucose is metabolised reductively according to pathway b):

$\mu_{S}^{f}=Y_{X / S}^{f} \cdot\left(q_{S}-q_{0}\right) \cdot \frac{Y_{X / O}^{r}}{Y_{X / S}^{r}}$.

Further, at fermentative conditions ethanol is produced and because of the lack of dissolved oxygen it cannot be consumed via pathway c); therefore:

$\mu_{E}^{r}=0$

\section{References}

1. Doran, P.M.; Bailey, J.E.: Effects of immobilization on growth, fermentation properties and macromolecular composition of Saccharomyces cerevisiae attached to gelatin. Biotech. Bioeng. 28 (1986) 73-87

2. Galazzo, J.L.; Bailey, J.E.: Growing Saccharomyces cerevisiae in calcium-alginate beads induces cell alterations which accelerate glucose conversion to ethanol. Biotech. Bioeng. 36 (1990) 417-426

3. Gikas, P.; Livingston, A.G.: Use of ATP to characterize biomass viability in freely suspended and immobilized cell bioreactors. Biotech. Bioeng. 42 (1993) 1337-1351

4. Kuriyama, H.; Ishibashi, H.; Miyagawa, H.; Kobayashi, H.; Eiichi, M.: Optimization of two-stage continuous ethanol fermentation using flocculating yeast. Biotech. Lett. 15 (1993) 415-420

5. Laplace, J.M.; Delgenes, J.P.; Moletta, R.; Navarro, J.M.: Ethanol production from glucose and xylose by separated and co-culture processes using high cell density systems. Process Biochem. 28 (1993) 519-525

6. Sousa, M.L.; Teixeira, J.A.: Reduction of diffusional limitations in yeast flocs. Biotech. Lett. 13 (1991) 883-888

7. Sousa, M.L.; Teixeira, J.A.; Mota, M.: Comparative analysis of ethanolic fermentation in two continuous flocculation bioreactors and effect of flocculation additive. Bioprocess Eng. 11 (1994) 83-90

8. Riley, M.R.; Muzzio, F.J.; Buettner, H.M.: Diffusion in heterogeneous media: application to immobilized cell systems. AIChE J. 41 (1995) 691-700

9. Riley, M.R.; Muzzio, F.J.; Buettner, H.M.; Reyes, S.C.: A simple correlation for predicting effective diffusivities in immobilized cell systems. Biotech. Bioeng. 49 (223-227) 1996
10. Webster, I.A.: Criteria for the prediction of diffusional control within whole cells and cell flocs. J. Chem. Tech. Biotechnol. 31 (1981) 178-182

11. Hamdi, M.: Biofilm thickness effect on the diffusion limitation in the bioprocess reaction: Biofloc critical diameter significance. Bioprocess Eng. 12 (1995) 193-197

12. Rieger, M.; Käppeli, O.; Fiechter, A.: The role of limited respiration in the incomplete oxydation of glucose by Saccharomyces cerevisiae. J. Gen. Microbiol. 129 (1983) 653-661

13. Sonnleitner, B.; Hahnemann, U.: Dynamics of the respiratory bottleneck of Saccharomyces cerevisiae. J. Biotechnol. 38 (1994) 63-79

14. Sonnleitner, B.; Käppeli, O.: Growth of Saccharomyces cerevisiae is controlled by its limited respiratory capacity: formulation and verification of a hypothesis. Biotech. Bioeng. 28 (1986) 927-937

15. Weir, S.; Ramdsen, D.K.; Hughes, J.; Le Thomas, F.: The strength of yeast flocs produced by the cationic flocculant chitosan: effect of suspension medium and of pretreatment with anionic polyelectrolytes. Biotechnol. Techniques 8 (1994) 129-132

16. Salt, D.E.; Bentham, A.C.; Hay, S.; Idris, A.; Gregory, J.; Hoare, M.; Dunnill, P.: The mechanism of flocculation of a Saccharomyces cerevisiae cell homogenate using polyethyleneimine. Bioprocess Eng. 15 (1996) 71-76

17. Miki, B.L.A.; Poon, N.H.; James, A.P.; Seligy, V.L.: Flocculation in Saccharomyces cerevisiae: mechanism of cell-cell interactions. In: Stewart, G.G.; Russel, I. (Eds.): Advances in Biotechnology: Current Developments in Yeast Research, pp. 193-198. Toronto: Pergamon Press 1981

18. Kuriyama, H.; Umeda, I.; Kobayashi, H.: Role of cations in the flocculation of Saccharomyces cerevisiae and discrimination of the corresponding proteins. Can. J. Microbiol. 37 (1991) 397-403

19. Mozes, N.; Schinckus, L.L.; Ghommidh, C.; Navarro, J.M.; Rouxhet, P. G.: Influence of medium composition on surface properties and aggregation of a Saccharomyces cerevisiae strain. Colloids and Surfaces B: Biointerfaces 3 (1994) 63-74

20. Lima, N.; Teixeira, J.A.; Mota, M.: Enhancement of metabolic rates of yeast flocculent cells through the use of polymeric additives. Bioprocess Eng. 7 (1992) 343-348

21. Sousa, M.L.; Teixeira, J.A.: Characterization of oxygen uptake and mass transfer in flocculent yeast cell cultures with or without a flocculation additive. Biotechnol. Lett. 18 (1996) 229-234

22. Vicente, A.A.; Dluhý, M.; Teixeira, J.A.: A new technique for measuring kinetic and mass transfer parameters in flocs of Saccharomyces cerevisiae. Biotechnol. Techniques 11 (1997) 113-116

23. Villadsen, J.; Michelsen, M.L.: Solution of Differential Equation Models by Polynomial Approximation. Englewood Cliffs, N. J.: Prentice-Hall 1978

24. Sousa, M.L.; Mota, M.; Teixeira, J.A.: Influence of operational parameters on the start-up of a flocculation airlift bioreactor. Colloids and Surfaces B: Biointerfaces 2 (1994) 181-188

25. Vicente, A.A.; Teixeira, J.A.: Hydrodynamic performance of a three-phase airlift bioreactor with an enlarged degassing zone. Bioprocess Eng. 14 (1995) 17-22 